\title{
Embarazo no deseado y fuentes de información impresas y audiovisuales, en mujeres peruanas (2004-2005)
}

\author{
Diana H uamán-Calderón ${ }^{1 a}$, Rocío Q uiliano-Terreros²b, \\ Carlos Vílchez-Román 3 a.
}

\section{Unwanted pregnancy and access to printed media in Peruvian women}

Background: It is possible that there is a relationship between reading newspapers and magazines, watching television and listening to the radio and unwanted pregnancies. Aim: To assess the relationship between the access to printed and audiovisual information resources and unwanted pregnancy in Peruvian women. Material and methods: A sample of 5,071 women, aged between 18 and 30 years was selected from a database of a Demographics and Health survey performed between 2004 and 2005. Using the information obtained in the survey, the association between unwanted pregnancies and reading newspapers and magazines, was analyzed. Results: Twenty six percent of women had at least an unwanted child $(\mathrm{n}=1,327)$. Radio and television were the media mostly used by these women. Seventy five percent of women almost never read newspapers and magazines. The lack of reading and the reliance in television and radio as sources of information were all associated with unwanted pregnancy ( $p<0.001$ ). Conclusions: In this sample of women, there is a negligible interest for reading and a high preference for radio and television. This explains the poor knowledge on sexual and reproductive health and the lack of impact of printed materials used in educational campaigns (Rev Méd Chile 2009; 137: 46-52).

(Key words: Multimedia; Pregnancy, unwanted; Sex education)

Recibido el 9 de enero, 2008. Aceptado el 6 de octubre, 2008.

${ }^{1}$ Organización Panamericana de la Salud (OPS), Lima, Perú. ${ }^{2}$ Biblioteca Central, Universidad San Martín de Porres (USMP), Lima, Perú. ${ }^{3}$ Organización por la Defensa de la Libertad Sexual (ORDELISE), Callao, Perú.

aLicenciado en Bibliotecología y Ciencias de la Información

bBachiller en Bibliotecología y Ciencias de la Información

$\mathrm{E}$ studios realizados en los últimos 15 años han revelado que existe una relación directa entre competencias lectoras y salud sexual y reproducti$\mathrm{va}^{6-8,12}$. Es decir, las personas con mejor desempeño lector tomarán mejores decisiones para evitar infecciones de transmisión sexual (ITS) y

Correspondencia a: Licenciado Carlos Vílchez-Román. Javier Luna Pizarro 132, Callao, Perú. Teléfono: (511) 5616323. Fax: (511) 4378289. E mail: carlos@ordelise.org. tendrán mayor efectividad en el empleo de métodos anticonceptivos.

En el Perú, la mayor parte del material de difusión sobre salud sexual y reproductiva es impreso, por ello es importante conocer el efecto de otros medios, como la radio y televisión. En el caso de las mujeres en edad fértil es fundamental, porque los indicadores demográficos muestran un aumento constante en el número de mujeres que tuvo al menos un hijo no deseado ${ }^{9,10}$. 
Antecedentes teóricos. Las principales fuentes de información empleadas por las personas para conocer más sobre un tema cualquiera, incluyendo la salud sexual y reproductiva, son los medios informativos tanto impresos como audiovisuales.

Según los datos del Censo Nacional de 2005, sólo $4 \%$ de mujeres que tienen entre 18 y 30 años son analfabetas, de allí la importancia de conocer cuál es su conocimiento en salud. En uno de los pocos trabajos locales sobre el uso de medios informativos por parte de mujeres en edad fértil, Sobero encuestó 288 mujeres del Callao, a fin de determinar sus conocimientos y fuentes de información sobre métodos anticonceptivos. Sus resultados fueron: $70 \%$ tiene un bajo nivel de conocimientos mientras que $31 \%$ alcanza un nivel medio. Con relación a las fuentes empleadas, $44 \%$ se informa a través de la obstetriz (matrona) y $35 \%$ consultando medios informativos como la radio y televisión ${ }^{16}$.

Como señalamos al inicio, los especialistas han estudiado la relación entre salud sexual y reproductiva, y el empleo de fuentes de información ${ }^{6-}$ 8,11,12. Ellos encontraron que el manejo adecuado del material impreso depende de las competencias lectoras y específicamente del llamado 'conocimiento de salud' (health literacy). Por lo tanto, las personas que tienen un bajo 'conocimiento de salud' no entenderán ni aprovecharán el material de promoción publicado y difundido.

Con relación al vínculo entre 'conocimiento de salud' y métodos anticonceptivos, Parker, Williams, Baker y Nurss señalan que la información disponible sobre cómo usar diversos métodos anticonceptivos es compleja, ya que está por encima del nivel de lectura promedio de los pacientes; los autores también comprueban que existe relación entre los embarazos no planificados y el logro académico $^{12}$. En 1999 Gazmararian, Parker y Baker examinaron la relación entre 'conocimiento de salud', uso de métodos anticonceptivos y planificación familiar. Para ello emplearon una versión abreviada del cuestionario TOFHLA (Test of Funcional Health Literacy in Adults). Los investigadores informan que $10 \%$ de las mujeres encuestadas tiene bajo 'conocimiento de salud'. Comparadas con las que tienen buen 'conocimiento de salud', las de bajo desempeño tienen mayor probabilidad de manejar información incorrecta sobre métodos anticonceptivos así como de haber tenido embarazos de alto riesgo ${ }^{8}$.
Posteriormente, Loraine Endres y cols aplicaron una versión abreviada del cuestionario TOFHLA en una muestra de 74 mujeres con diabetes pregestacional, procedentes del área de Chicago, Estados Unidos de Norteamérica y encontraron que 22\% tiene bajo 'conocimiento de salud'. Dichas mujeres tienen mayor probabilidad de tener embarazos no planificados $(\mathrm{p}=0,002)$ y mucha menor probabilidad de informarse con un endocrinólogo u obstetriz $(\mathrm{p}=0,01)^{6}$. Desde una perspectiva diferente, Margaret Freda y su equipo de colaboradoras analizaron la legibilidad de 100 folletos -escritos en inglés- sobre educación en ginecología y obstetricia. Para ello trabajaron con cuatro fórmulas de legibilidad de textos, incluyendo la de Flesh, Gunning y el SMOG de McLaughlin. De acuerdo con sus resultados, el material de divulgación está escrito en un nivel de legibilidad por encima del recomendado para el público en general ${ }^{7}$.

Los resultados obtenidos al medir la legibilidad de los textos y aplicar pruebas estandarizadas de 'conocimiento de salud' revelan la magnitud del impacto negativo que estas carencias producen en la prevención del embarazo no deseado, tanto en el tiempo y dinero invertido por cada persona, como en los recursos asignados por el sistema de salud pública y en su capacidad de atención. Los datos existentes muestran que los conocimientos limitados sobre salud sexual y reproductiva, por parte de las mujeres en edad fértil, influyen en la efectividad de los métodos anticonceptivos empleados. En lo que se refiere a las fuentes de información no impresas, los investigadores locales aún no han abordado la relación entre embarazo no deseado y el consumo de radio y televisión porque no hay campañas informativas, de alcance nacional, sobre salud sexual y reproductiva. La mayoría de programas de entrevistas y consultorios médicos sobre salud sexual y reproductiva son esporádicos y aquellos que abordan la sexualidad desde una perspectiva más amplia son censurados.

$\mathrm{Si}$ bien es cierto no hay estudios que exploren dicha relación, existe un trabajo que identifica los factores sociales y demográficos que influyen en la salud sexual y reproductiva de las mujeres jóvenes. Por ejemplo, los investigadores encontraron que la brecha entre mujeres adolescentes y jóvenes, en lo que se refiere al inicio sexual (temprano o tardío), frecuencia de actividad 
sexual, situación afectiva y embarazo (deseado y no deseado) era explicada por la edad, alfabetismo, educación, trabajo, unión, región, etnicidad y localidad. Los autores informaron que $64 \%$ de adolescentes gestantes, semialfabetas y con primaria incompleta y $55 \%$ de jóvenes, analfabetas rurales y convivientes, tuvo embarazos no desea$\operatorname{dos}^{13}$. Tal como lo mencionamos al inicio, nuestro objetivo fue determinar si la lectura de diarios y revistas y el consumo habitual de radio y televisión tiene una relación de concurrencia simultánea con el embarazo no deseado. En ese sentido sometimos a prueba el siguiente modelo teórico:

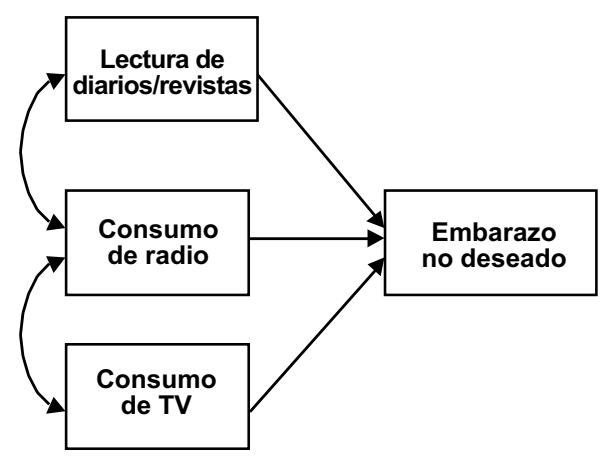

De acuerdo con nuestro modelo, las tres variables asociadas al embarazo no deseado están relacionadas entre sí porque estudios previos ${ }^{13}$ han mostrado que la escolaridad es un factor asociado al embarazo no deseado, y dentro de ésta uno de los aspectos centrales es la capacidad para manejar información, sea impresa o audiovisual. Aquí es importante hacer una aclaración: el gráfico mostrado no representa un diagrama de ruta (elemento necesario para llevar a cabo un análisis de ruta), sino que a través de él hemos resaltado que existe una relación entre las variables propuestas en el modelo.

\section{MATERIAL Y MÉTODO}

Empleamos la base de datos de la Encuesta Nacional Demográfica y de Salud Familiar (Endes), correspondiente a los años 2004 y 2005. Dicha encuesta tiene como objetivo obtener datos sobre salud materno-infantil, nutrición, los niveles de fecundidad, el conocimiento y uso de métodos de planificación familiar, incluyendo las fuentes utilizadas para acceder a ellos, ITS y VIH/SIDA ${ }^{10}$.

Endes es una encuesta que se aplica periódicamente a mujeres mayores de 15 años por el Instituto Nacional de Estadística e Informática (Inei). Sus resultados son un insumo valioso para la formulación de políticas de salud pública y campañas de prevención llevadas a cabo por el Ministerio de Salud. Anteriormente la encuesta fue aplicada en 1986, 1991/1992, 1996 y 2000.

De acuerdo con el registro de la base de datos Endes 2004-2005, 12.465 mujeres participaron en el estudio. Para la presente investigación, a través del programa SPSS seleccionamos una muestra de 5.071 mujeres. El criterio de selección sólo consideró mujeres con una edad igual o mayor a 18 años y menor o igual a 30 años. Elegimos este rango de edad porque corresponde con los años de mayor natalidad en las mujeres. Aun cuando desde un punto de vista obstétrico, las adolescentes menores de 18 años constituyen un grupo de alto riesgo, dicha población no fue considerada en el estudio porque la problemática de este grupo etáreo ya fue analizado en un estudio similar ${ }^{18}$.

Las encuestadas menores de 18 o mayores de 30 fueron excluidas de la muestra. Debido a su alcance nacional, las mujeres incluidas en esta investigación procedían de todas las regiones del Perú. En cuanto al análisis de datos, empleamos el estadístico $\chi^{2}$, el cual permitió determinar si existe asociación entre las variables incluidas en el modelo. El coeficiente $\chi^{2}$ de Pearson es útil para comparar la frecuencia de casos de una variable nominal en dos o más muestras independientes (en esta investigación, una muestra de mujeres que desearon tener su último hijo y aquéllas que no lo desearon) y luego determinar el nivel de significancia estadística.

Nuestro modelo consideró las siguientes variables: lectura de diarios y revistas, consumo de radio, consumo de televisión y embarazo no deseado. La lectura de diarios y revistas fue considerada como una sola variable porque la encuesta Endes, de donde hemos tomado los datos, ya los tenía codificados de esa manera; ésta es una de las limitaciones de nuestra investigación. Para la discusión de los datos, seleccionamos las variables que alcanzaron significancia estadística $(p<001)$. De acuerdo con el diseño de investigación, este es un estudio descriptivo con alcance exploratorio. 


\section{RESULTADOS}

El promedio de edad de las encuestadas fue 23,71 $\pm 3,79$ años, $6,1 \%$ informó estar actualmente embarazada ( $\mathrm{n}=310)$ y $57 \%$ manifestó ser madre de uno o más hijos ( $\mathrm{n}=2.905)$, cuatro de ellas dijeron tener 7 hijos y una encuestada señaló que tenía 8 hijos. En lo que se refiere al uso de medios informativos, $75 \%$ dijo casi no leer diarios 0 revistas, mientras que $25 \%$ lee ocasionalmente o todos los días. Cuando comparamos el consumo de radio y televisión encontramos la situación contraria: casi $70 \%$ informó escuchar radio ocasionalmente 0 todos los días, en cuanto a la televisión el consumo ocasional o frecuente llegó a $59 \%$ (Figura 1).
Las mujeres que no desearon tener su último hijo (o que no lo desearon al momento de quedar embarazadas), mostraron preferencias similares en líneas generales, aunque con una ligera disminución en el número que leen diarios y revistas menos de una vez por semana y un incremento considerable en la proporción de aquellas que nunca leen, oyen radio ni ven televisión (Figura 2).

En lo que se refiere al análisis de asociación con la prueba $\chi^{2}$, comprobamos que la lectura de diarios y revistas, consumo de radio y televisión tuvieron una relación de concurrencia simultánea ( $p$ <.001) y que las tres variables del modelo estuvieron relacionadas con el embarazo no deseado (Tablas 1 y 2); sin embargo ninguna de ellas fue contundente. Los datos obtenidos al analizar

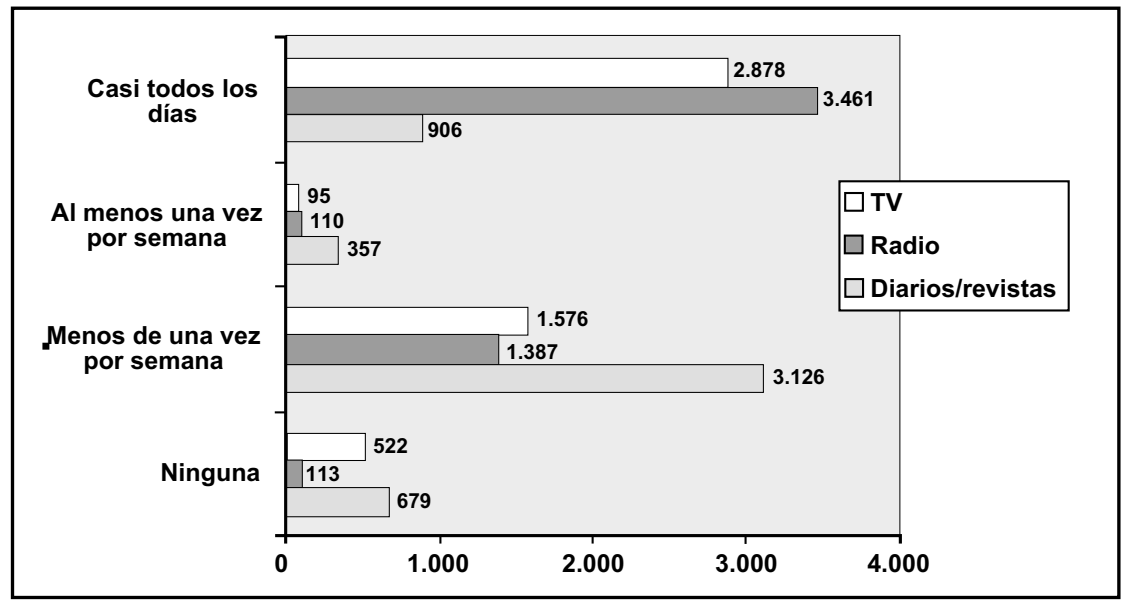

Figura 1. Fuentes informativas empleadas por las mujeres encuestadas.

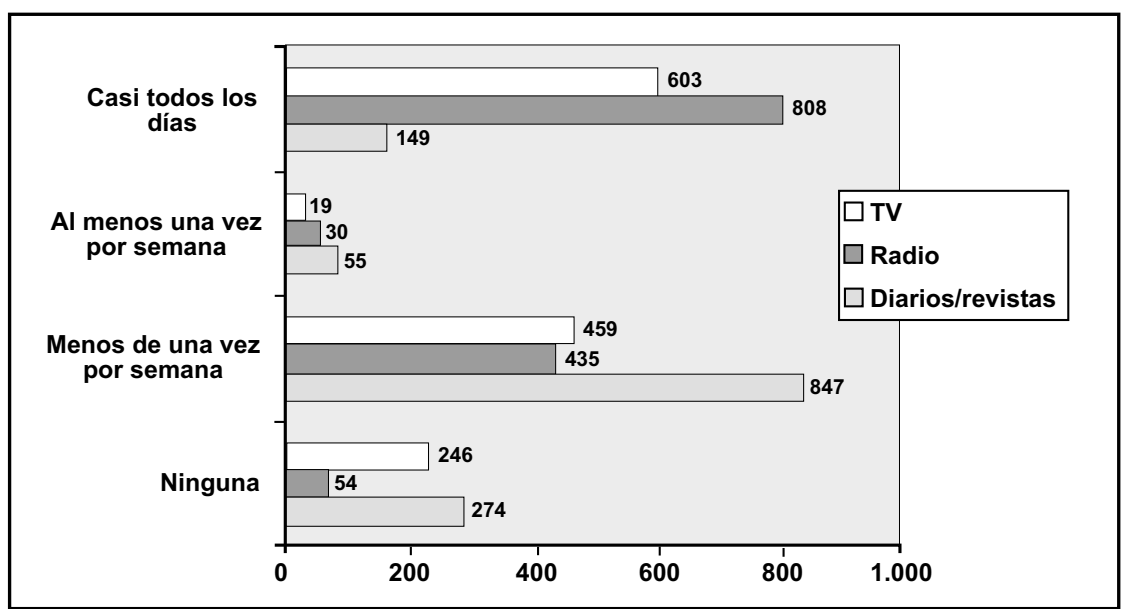

Figura 2. Fuentes informativas de las mujeres que tuvieron embarazos no deseados. 
Tabla 1. M atriz de asociación entre las variables del modelo

\begin{tabular}{|lccc|}
\hline Variables & $\begin{array}{c}\text { Lectura de } \\
\text { diarios y revistas }\end{array}$ & $\begin{array}{c}\text { Consumo } \\
\text { de radio }\end{array}$ & $\begin{array}{c}\text { Consumo } \\
\text { de televisión }\end{array}$ \\
\hline $\begin{array}{l}\text { Lectura de diarios y revistas } \\
\text { Consumo de radio }\end{array}$ & -- & & \\
Consumo de televisión & $1.146 .90^{*}$ & - & - \\
\hline
\end{tabular}

*Nivel de significancia $\mathrm{p}<001$.

Tabla 2. Asociación de las variables del modelo con el embarazo no deseado

\begin{tabular}{|lcc|}
\hline Variables & $\chi^{2}$ de Pearson & Nivel de significancia \\
\hline Lectura de diarios y revistas & 57.094 &, $001 *$ \\
Consumo de radio & 24.038 &, $001^{*}$ \\
Consumo de televisión & 108.826 &, $001 *$ \\
\hline
\end{tabular}

la Encuesta Endes Continua 2004-2005 muestran que el modelo planteado era correcto.

Dado que se trata de variables nominales, no pudimos establecer una relación de causalidad, como sí ocurre cuando se emplean modelos de regresión. Sobre este punto, es necesario recordar que la evidencia de una asociación entre variables no es prueba de causalidad, porque para ello necesitaríamos incluir en el análisis otras variables que no fueron consideradas en el modelo teórico.

\section{Discusión}

Los resultados confirman la tendencia ya observada en otros estudios: la preferencia casi absoluta por los programas de radio y televisión está asociada con el desinterés generalizado por la lectura de libros, revistas y textos en general, a pesar que los diarios y revistas tienen precios asequibles a la mayoría de la población. Incluso el concepto mismo de lectura ha cambiado, ello explica que muchas personas consideren que ver los titulares de la portada, mirar la sección de historietas o apreciar las fotos de la sección deportes y espectáculos, equivale a leer un diario ${ }^{2,14}$.
Las encuestas nacionales tampoco ayudan mucho porque presentan fallas en el diseño metodológico. Por ejemplo, el año 2004 la Biblioteca Nacional del Perú y la Universidad Nacional de Ingeniería encuestaron a 19.968 personas para conocer el hábito de lectura de los peruanos, y encontraron que ante la pregunta "¿Le gusta leer?" $90 \%$ señaló que sí le gusta leer ${ }^{17}$. Obviamente, casi todos iban a responder que sí porque no está bien visto socialmente que digamos que no nos gusta la lectura. Y es que no es coherente que 90\% diga que sí le gusta la lectura, pero que casi $60 \%$ señale que no lee libros y que sólo $12 \%$ manifieste haber leído tres o más libros en los tres meses anteriores a la encuesta.

Como ya mencionamos, este rechazo por la lectura está asociado con un consumo creciente de programas de radio y televisión. Este hecho resulta preocupante no sólo porque el predominio de la cultura audiovisual supone una pérdida del análisis crítico y la capacidad creativa (dado que las ideas y conceptos ya vienen 'empaquetados' y listos para ser consumidos por los televidentes y radioescuchas $)^{5}$, sino porque el tratamiento de la salud sexual y reproductiva se reduce a un tema de métodos anticonceptivos e infecciones de transmisión sexual y no a una educación sexual 
saludable. Como mencionamos antes, cuando los programas mantienen una campaña permanente a favor de la educación sexual, suelen ser sacados del aire por la presión de los grupos conservadores y la Iglesia Católica.

Para hacer frente a este problema necesitamos atacarlo frontalmente a través de una adecuada articulación del programa de educación sexual que debiera contar con todo el apoyo de los Ministerios de Educación y de Salud, brindando atención directa a la población, servicios de información y orientación enmarcados dentro de programas nacionales de mayor alcance. También es necesario evaluar con herramientas de recolección y análisis, como las pruebas estandarizadas sobre 'conocimiento de salud', si el público entiende el contenido del material difundido de los programas de educación sexual, las cuales serán de gran utilidad en los establecimientos de salud, públicos y privados, del país.

En conclusión hemos comprobado que existe relación entre las variables propuestas en el modelo y el embarazo no deseado. Para profundizar más en este tema debemos desarrollar modelos causales que tengan en cuenta otras variables explicativas, como las sociales y económicas, que también influyen en el embarazo no deseado o también estudios de corte cualitativo y exploratorio. Si no trabajamos por mejorar el 'conocimiento de salud' en temas específicos como salud sexual y reproductiva, ni brindamos facilidades para el acceso a fuentes de información confiables, cada año se presentarán nuevos casos de embarazos no deseados, los cuales tienen un gran impacto negativo en la calidad de vida de la población, ya que supone dividir los pocos ingresos familiares entre un mayor número de personas. Las campañas de promoción y los programas deberán estar dirigidos a hombres y mujeres, porque ambos son responsables de la planificación familiar, aquí el reto está en incorporar a los varones. El otro gran reto es darle cada vez más importancia a los

\section{REFERENCIAS}

1. Aguirre A, Ebrahim N, Shea J. Performance of the English and Spanish S-TOFHLA among publicly insured Medicaid and Medicare patients. Patient Educ Couns 2005; 56: 332-9. materiales audiovisuales, como parte del material de difusión elaborado por los comunicadores sociales dedicados a la promoción de la salud.

Teniendo en cuenta lo antes señalado, para enfrentar los casos de embarazos no deseados, los autores presentamos dos alternativas que permitirán reducirlos progresivamente:

1. Identificar el nivel actual del 'conocimiento de salud' de las mujeres en edad fértil a través del empleo de pruebas estandarizadas. Aun cuando varios especialistas han trabajado con la versión abreviada del TOFHLA (Test of Funcional Health Literacy in Adults), cuestionario originalmente desarrollado en inglés y que cuenta con una versión en español ${ }^{1,4,8,12}$, existe otra herramienta creada específicamente para las personas que hablan español: el SAHLSA-50 (Short Assessment of Health Literacy in Spanish-speaking adults), el cual también tiene altos niveles de validez y confiabilidad y cuya aplicación demora entre 12 y $15 \mathrm{~min}^{15}$. Por ello, los autores del presente estudio validarán el cuestionario SAHLSA-50 y analizarán las propiedades psicométricas en un estudio que se publicará próximamente.

2. Implementar una red de centros de asesoramiento sexual (CAS) en el teritorio nacional. Los CAS serán proyectos de desarrollo social cuya viabilidad será analizada a través del Sistema Nacional de Inversión Pública (SNIP). Estos centros contarán con profesionales de la educación y salud (educadores, enfermeras, psicólogos, médicos y orientadores) quienes asesorarán y orientarán a las personas que acudan a dichos centros. Además, contarán con equipos audiovisuales y material (impreso, en audio y video) sobre temas relacionados con la salud sexual y reproductiva. De esta manera, los usuarios de cada centro complementarán la explicación recibida por el equipo profesional con el material disponible en cada establecimiento y los videos educativos encontrados en internet.

2. Alfaro R, Macassi S. Padres e hijos frente la pantalla. En: Alfaro R (ed.). Seducidos por la tele. Lima: Asociación de Comunicadores Sociales Calandria; 1995; 87-139.

3. Apoyo Opinión y Mercado. Perfil del adolescente y del joven 2006. Lima: Apoyo; 2006. 
4. Baker D, Wiluams M, Parker R, Gazmararian J, Nurss J. Development of a brief test to measure functional health literacy. Patient Educ Couns. 1999; 38: 33-42.

5. Bordieu P. Sobre la televisión. Barcelona: Anagrama; 1997.

6. Endres L, Sharp L, Haney E, Dooley S. Health literacy and pregnancy preparedness in pregestational diabetes. Diabetes Care 2004; 27: 331-4.

7. Freda M, Damus K, Merkatz I. Evaluation of the readability of ACOG patient education pamphlets. Obstet Gynecol 1999; 93: 771-4.

8. Gazmararian JA, Parker RM y BaKer DW. Reading skills and family planning knowledge and practices in a low-income managed-care population. Obstet Gynecol 1999; 93: 239-44.

9. Instituto Nacional de Estadística e Informática. Encuesta demográfica y de salud familiar, Endes 2000. Lima: Inei; 2001.

10. Instituto Nacional de Estadística e InFormática. Encuesta demográfica y de salud familiar 2004-2005 Endes continua: informe final. Lima: Inei; 2006.

11. Osborne H. Health Literacy from A to Z: Practical ways to communicate your health message. Sudbury, Massachusetts: Jones and Bartlett; 2005.

12. Parker RM, Wiluams MV, Baker DW, Nurss JR. Literacy and contraception: Exploring the link. Obstet Gynecol 1996; 88 (3 Suppl.): 72S-77S. [Resumen]
13. Raguz M. Salud sexual y reproductiva adolescente y juvenil: condicionantes sociodemográficos e implicancias para políticas, planes y programas e intervenciones. Lima: Centro de Investigación y Desarrollo; Instituto de Estadística e Informática; 2002.

14. Santos Anaya M. ¿Cuán libre es el tiempo "libre" de los jóvenes limeños? Flecha Azul 2000; 13: 20-7.

15. Shoou-Yih D, Bender D, Ruiz R, Young C. Development of an easy-to-use Spanish health literacy test. Health Serv Res 2006; 41: 1392-412.

16. Sobero García MR. Nivel de conocimiento y fuentes de información sobre métodos anticonceptivos que tienen las mujeres en edad fértil del Pueblo Joven Aeropuerto - Callao. [Tesis de Licenciatura] Lima: Facultad de Obstetricia y Enfermería, Universidad San Martín de Porres; 1994.

17. Universidad Nacional de Ingeniería. Hábitos de lectura y ciudadanía informada en la población peruana. Lima: Biblioteca Nacional del Perú; 2004.

18. Romaní ZegarRa S, Vílchez-Román C. Embarazo, hábitos de lectura y consumo de radio y televisión en adolescentes peruanas: explorando relaciones de asociación. ACIMED 2008; 14 (4). Disponible en http://scielo.sld.cu/scielo.php?script=sci_arttext\& pid =S1024-94352008000400008\&lng=es\&nrm=iso . [Consultado: 20/06/2008]. 